\title{
Role of RANK ligand in mediating increased bone resorption in early postmenopausal women
}

\author{
Guitty Eghbali-Fatourechi, ${ }^{1}$ Sundeep Khosla, ${ }^{1}$ Arunik Sanyal, ${ }^{1}$ William J. Boyle, ${ }^{2}$ \\ David L. Lacey, ${ }^{2}$ and B. Lawrence Riggs ${ }^{1}$
}

${ }^{1}$ Endocrine Research Unit, Mayo Clinic and Foundation, Rochester, Minnesota, USA

${ }^{2}$ Department of Metabolic Disorders, Amgen Inc., Thousand Oaks, California, USA

\begin{abstract}
Studies in rodents have implicated various cytokines as paracrine mediators of increased osteoclastogenesis during estrogen deficiency, but increases in RANKL, the final effector of osteoclastogenesis, have not been demonstrated. Thus, we isolated bone marrow mononuclear cells expressing RANKL on their surfaces by two-color flow cytometry using FITC-conjugated osteoprotegerin-Fc (OPG-Fc-FITC) as a probe. The cells were characterized as preosteoblastic marrow stromal cells (MSCs), T lymphocytes, or B lymphocytes by using Ab's against bone alkaline phosphatase (BAP), CD3, and CD20, respectively, in 12 premenopausal women (Group A), 12 early postmenopausal women (Group B), and 12 age-matched, estrogen-treated postmenopausal women (Group C). Fluorescence intensity of OPG-Fc-FITC, an index of the surface concentration of RANKL per cell, was increased in Group B over Groups A and C by two- to threefold for MSCs, T cells, B cells, and total RANKL-expressing cells. Moreover, in the merged groups, RANKL expression per cell correlated directly with the bone resorption markers, serum C-terminal telopeptide of type I collagen and urine $\mathrm{N}$-telopeptide of type I collagen, in all three cell types and inversely with serum $17 \beta$-estradiol for total RANKL-expressing cells. The data suggest that upregulation of RANKL on bone marrow cells is an important determinant of increased bone resorption induced by estrogen deficiency.
\end{abstract}

J. Clin. Invest. 111:1221-1230 (2003). doi:10.1172/JCI200317215.

\section{Introduction}

Osteoporosis is a major metabolic bone disease that over a lifetime results in fractures in $40 \%$ of aging women and $15 \%$ of aging men (1). Recent studies have shown that estrogen deficiency is the cause of both the early and the late forms of osteoporosis in postmenopausal women and contributes to the development of osteoporosis in aging men $(1,2)$. Estrogen deficiency is associated with an increase in bone resorption over bone formation, leading to excessive and sustained bone loss. The increase in bone resorption is due both to increased osteoclastogenesis and to decreased osteoclast apoptosis $(1,3)$. Since the demonstration in 1988 that bone cells contain functional estrogen receptors

Received for publication October 22, 2002, and accepted in revised form February 4, 2003.

Address correspondence to: B. Lawrence Riggs, Mayo Clinic, 200 First Street SW, North 6 Plummer, Rochester, Minnesota 55905, USA. Phone: (507) 284-3961;

Fax: (507) 284-8271; E-mail: riggs.lawrence@mayo.edu.

William J. Boyle's present address is: Protein Pathways Inc.,

Woodland Hills, California, USA.

Conflict of interest: The authors have declared that no conflict of interest exists.

Nonstandard abbreviations used: receptor activator of NF- $\kappa B$ (RANK); RANK ligand (RANKL); marrow stromal cells (MSCs); osteoprotegerin (OPG); bone alkaline phosphatase (BAP); phycoerythrin (PE); C-terminal telopeptide of type I collagen (CTx); coefficient of variation (CV); N-telopeptide of type I collagen (NTx).
(1), the primary focus of research has been to identify the paracrine mediators of the increased osteoclastogenesis induced by estrogen deficiency.

Despite wide-ranging studies, the identity of the paracrine mediators of bone loss induced by estrogen deficiency is still unclear. Studies in ovariectomized rodents and in osteoblastic cell lines in vitro have implicated increases in various proinflammatory cytokines - IL-1 $\beta$, IL-6, TNF- $\alpha$, GM-CSF, MCSF, and PGE $_{2}$ (see reviews; refs. $1,3,4)$. These cytokines increase bone resorption mainly by increasing the pool size of preosteoclasts in bone marrow $(1,3,4)$; however, MCSF also enhances fusion of preosteoclasts to form osteoclasts (5) and, recently, direct effects of TNF- $\alpha$ on osteoclasts have been demonstrated $(6,7)$. Measures that impair the synthesis of or the response to IL- $1 \beta$, IL-6, TNF- $\alpha$, or $\mathrm{PGE}_{2}$ attenuate or prevent ovariectomy-induced bone loss in rodents $(1,3,4)$. In addition, estrogen upregulates TGF- $\beta$ (8), an inhibitor of bone resorption that decreases the activity and increases the rate of apoptosis of mature osteoclasts (9).

All of these earlier findings, however, must now be reevaluated in the wake of the recent identification of three new members of the TNF ligand and receptor-signaling family that are the final effectors of bone resorption (see reviews ; refs. 10-12). RANK ligand (RANKL) is both necessary and sufficient for osteoclast differentiation, provided that permissive concentrations of MCSF are present, and also enhances activity and prolongs the 
lifespan of osteoclasts by decreasing apoptosis. In bone marrow, RANKL is expressed on the surfaces of MSCs of the osteoblast lineage (13), T lymphocytes (6), and B lymphocytes (14). Cell-to-cell contact allows RANKL to bind to its physiologic receptor RANK, which is expressed on the surface of osteoclast lineage cells. Its action is opposed by osteoprotegerin (OPG), a neutralizing soluble decoy receptor, produced by MSCs and osteoblasts.

Because of its pivotal role in osteoclast formation and function, the RANKL/RANK/OPG regulatory system is likely to be involved in the antiresorptive action of estrogen. Indeed, estrogen has been demonstrated to upregulate gene expression and protein synthesis of OPG, both in vitro in human (15) and in rodent (16) osteoblastic cells and in vivo in elderly men (17). In contrast, previous studies have not demonstrated that either RANKL (16) or RANK (18) expression or activity is regulated by estrogen. Moreover, no studies have as yet been reported on RANKL expression made in human bone marrow cells.

The objective of our study was to determine if RANKL plays a role in mediating the increase in bone resorption in early postmenopausal women and, thus, in the pathogenesis of postmenopausal osteoporosis. To address this issue, we have developed a method for isolating cells expressing RANKL on their surface from human bone marrow by dual-color flow cytometry using the FITC-labeled OPG-Fc fusion protein as a fluorescent probe for cells. With this new method, we find that bone marrow cells from untreated early postmenopausal women have increased surface expression of RANKL as compared with estrogen-replete women. We further demonstrate that the increased surface expression of RANKL on bone marrow cells correlates directly with increases in bone resorption markers and inversely with serum $17 \beta$-estradiol levels.

\section{Methods}

Study subjects and experimental protocol. We studied 36 healthy volunteer women, including 12 premenopausal women (Group A), 12 untreated postmenopausal women (Group B), and 12 postmenopausal receiving estrogen-replacement therapy (Group C) (Table 1). The subjects in Group $\mathrm{C}$ were changed from their usual estrogen-replacement regimen to $0.1 \mathrm{mg}$ estradiol patches, applied twice weekly, for 60 days prior to bone marrow aspiration, and the accompanying progestin, if present, was discontinued. For inclusion, we required good health and the absence of any serious acute or chronic medical conditions, including blood dyscrasias and medical conditions or drug use that might affect bone or calcium metabolism or alter bone marrow function. We also excluded premenopausal women who were receiving oral contraceptives. We obtained protocol approval by the Mayo Institutional Review Board and written, informed consent from the study subjects.

Studies were performed as outpatients at the Mayo General Clinical Research Center. A fasting blood sample was drawn at 0800 hours, and a second morning voided urine sample was obtained for biochemical measurements. Samples were stored at $-80^{\circ} \mathrm{C}$ until analyzed. Under local anesthesia, a total of $20 \mathrm{ml}$ of bone marrow aspirate was collected on EDTA from both iliac crests using a standard procedure.

Reagents. Ficoll-Paque Plus was purchased from Amersham Pharmacia Biotec AB (Uppsala, Sweden). Normal goat serum and the phycoerythrin-conjugated secondary $\mathrm{Ab}$, goat anti-mouse $\mathrm{Ab}$, and human Fc-fragment conjugated to FITC were purchased from Jackson ImmunoResearch Laboratories Inc. (West Grove, Pennsylvania, USA). We obtained mAb's B4-78 for human bone alkaline phosphatase (BAP), a mouse monoclonal $\operatorname{IgG}_{1}$, and STRO-1, a mouse IgM, from the Developmental Studies Hybridoma Bank, University of Iowa (Iowa City, Iowa, USA). The secondary Ab's were goat anti-mouse IgG conjugated to phycoerythrin (PE). Monoclonal mouse IgG $_{2 a}$ anti-human CD20 for B cells, clone B9E9, monoclonal mouse IgG $_{1}$ anti-human CD3 for T cells, clone UCHT-1, isotype controls, including mouse $\operatorname{IgG}_{1 \kappa}\left(\right.$ MOPC-21), mouse $\operatorname{IgG}_{2 \mathrm{a}, \mathrm{\kappa}}$ (UPC-10), and mouse IgG 2 b, were purchased from Sigma-Aldrich (St. Louis, Missouri, USA). The anti-human granulocyte $\mathrm{CD} 15 \mathrm{Ab}$ was a monoclonal mouse IgM isotype clone G155-228 from BD PharMingen (San Diego, California, USA). Anti-TRAIL mAb was a mouse IgG $_{1}$ from R\&D Systems Inc. (Minneapolis, Minnesota, USA). OPG-Fc was a recombinant human fusion protein produced in CHO cells in which the N-terminal ligandbinding domain (amino acid residues 22-194) of OPG

Table 1

Clinical characteristics and biochemical measurements (mean \pm SEM)

\begin{tabular}{|c|c|c|c|c|c|c|c|}
\hline Variable & Group A & Group B & Group C & $P^{\mathrm{A}}$ & $A$ vs. $B^{B}$ & A vs. $C^{B}$ & B vs. $C^{-}$ \\
\hline$n$ & 12 & 12 & 12 & - & - & - & - \\
\hline Age $(y r)$ & $31.8 \pm 5.8$ & $53.8 \pm 3.4$ & $52.3 \pm 3.5$ & - & - & - & - \\
\hline Last menstrual period $(\mathrm{yr})$ & - & $3.1 \pm 1.5$ & $3.3 \pm 1.5$ & - & - & - & - \\
\hline Serum CTx $(\mathrm{pmol} / \mathrm{l})$ & $4,366 \pm 669$ & $6,119 \pm 576$ & $3,071 \pm 533$ & $<0.001$ & $<0.05$ & NS & $<0.05$ \\
\hline Urine NTx $(\mathrm{nmol} / \mathrm{mmol} \mathrm{Cr})$ & $36 \pm 4.1$ & $52 \pm 5.42$ & $27 \pm 4.8$ & $<0.01$ & $<0.05$ & NS & $<0.05$ \\
\hline Serum $E_{2}(p m o l / l)$ & $164.8 \pm 30.5$ & $40.7 \pm 5.9$ & $208.5 \pm 3.3$ & $<0.001$ & $<0.05$ & NS & $<0.05$ \\
\hline Serum OPG (pmol/l) & $2.05 \pm 0.35$ & $1.80 \pm 0.32$ & $1.60 \pm 0.15$ & NS & NS & NS & NS \\
\hline Serum RANKL(pmol/l) & $0.84 \pm 0.24$ & $1.37 \pm 0.68$ & $1.55 \pm 0.99$ & NS & NS & NS & NS \\
\hline
\end{tabular}

${ }^{A}$ ANOVA; ${ }^{B}$ Student-Neuman-Keuls multiple comparison test. $\mathrm{Cr}$, creatinine. 

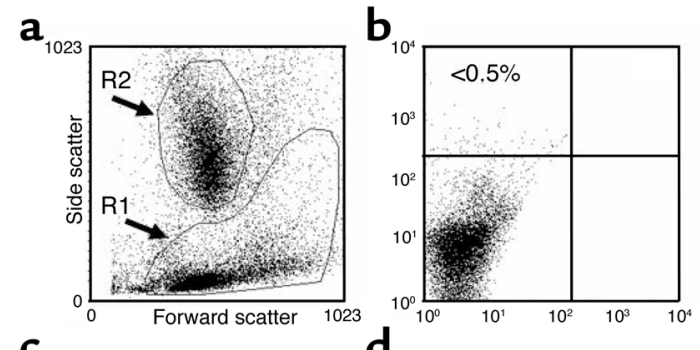

C
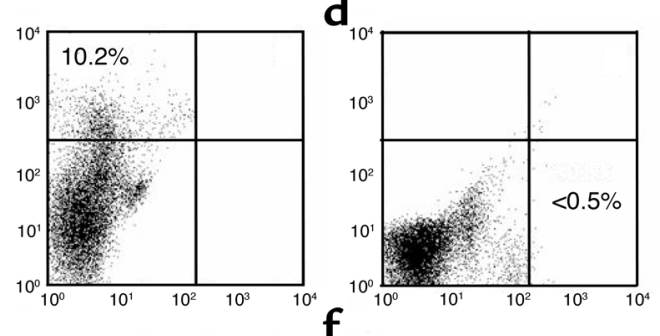

e

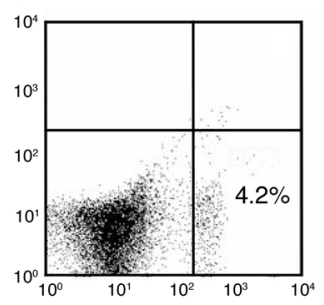

for

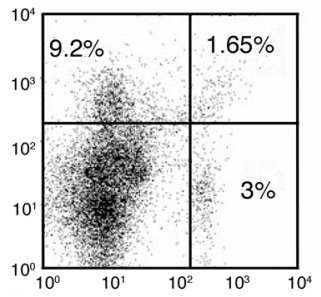

had been fused to the glutamic acid at residue 211 of the human $\mathrm{Fc}(19)$. This region was conjugated to the FITC (19). The resulting OPG-Fc fusion protein lacks the $\mathrm{C}$-terminal heparin-binding domain and bound to cells in a RANKL-dependent manner (20). StrataPrep Total RNA Miniprep Kit was from Stratagene (La Jolla, California, USA). Reverse transcriptase, avian myeloblastosis virus, primer random $(\mathrm{dN})_{6}$, RNase inhibitor, dNTPs, and LightCycler-Fast Start DNA Master Syber Green were from Roche Molecular Biochemicals (Mannheim, Germany).

Cell preparation and flow cytometry. Bone marrow aspirate $(7.5 \mathrm{ml})$ was diluted 1:2 in PBS ( $\mathrm{vol} / \mathrm{vol})$, layered over a Ficoll-Paque density gradient, centrifuged, and bone marrow mononuclear cells were collected. These were washed, counted with a hemocytometer, and cell viability was determined by trypan blue exclusion. After exposure to $10 \%$ normal goat serum to block nonspecific binding sites, aliquots of $100 \mu \mathrm{l}\left(\sim 10^{6}\right.$ cells) were incubated for 30 min with primary Ab's against BAP, CD3, or CD20, washed, and incubated again for $30 \mathrm{~min}$ with secondary $\mathrm{Ab}$ 's conjugated to $\mathrm{PE}$, then washed, and finally incubated with the OPG-Fc-FITC probe at $4^{\circ} \mathrm{C}$ for $60 \mathrm{~min}$.

For the analysis of flow cytometry data, we initially used forward/side light scatter to set regions around the lymphocyte/monocyte-enriched area (R1 region) and around the granulocyte-enriched area ( $\mathrm{R} 2$ region) (Figure 1a). The R1 region contained the cell populations of interest - MSC, T lymphocytes, and B lymphocytes - as well as monocytes. The gates were set to select and analyze the R1 region and to exclude the R2

\section{Figure 1}

Representative flow cytometry dot plots sorted for $\mathrm{BAP}^{+} / \mathrm{RANKL}^{+}$ cells from bone marrow aspirates of an untreated postmenopausal woman. (a) Forward/side light scatter profile of unstained cells following Ficoll-Paque density centrifugation. The R1 gate represents the mononuclear cells that include the MSCs. The R2 gate represents granulocyte precursor cells and these are excluded from analysis. (b) The dot plot profile of the autofluorescence from the irrelevant mouse $\lg G_{1}$ isotype that was used as a control for anti-BAP Ab. (c) The dot plot profile of the $\mathrm{BAP}^{+}$cells (left upper quadrants in $\mathbf{a}-\mathbf{f}$ ) as assessed by single-color flow cytometry using the monoclonal antiBAP (B4-78) stained with a PE-conjugated secondary Ab. The quadrant limits were set so that the left upper quadrant that defines the specific fluorescence for the $\mathrm{BAP}^{+}$cells contains less than $5 \%$ of the nonspecific fluorescence from the irrelevant mouse $\lg _{1}$ isotype control. (d and $\mathbf{e}$ ) The analogous single-color flow cytometry using the human Fc-FITC control and OPG-Fc-FITC probe for RANKL ${ }^{+}$sorted cells, respectively. (f) The dual-color dot plot profile for $\mathrm{BAP}^{+} / \mathrm{RANKL}^{-}$sorted cells (left upper quadrant), $\mathrm{BAP}^{-} / \mathrm{RANKL}^{+}$sorted cells (right lower quadrant), and for combined $\mathrm{BAP}^{+}$and $\mathrm{RANKL}^{+}$ sorted cells (right upper quadrant). The numerical percentages represent the stained cells per quadrant expressed as a proportion of total mononuclear cells in the R1 gate.

region. Positive populations were identified as cells that expressed specific levels of fluorescence activity above the nonspecific autofluorescence of the isotype control. Quadrants in samples were set so that the same quadrant in the control included positive cells at a proportion less than $5 \%$ of that of samples with specific positive fluorescence for a signature marker. Cells identified by single- or dual-color flow cytometry were expressed as a percentage of gated cells on R1. With the same analysis, we assessed mean fluorescence intensity of OPG-Fc-FITC per cell, which is an index of the surface concentration of RANKL molecules.

\section{Table 2}

Primers used for the RT-PCR analysis

Gene Primers

$A P$

Sense: 5'-TCAAACCGAGATACAAGCAC-3' Antisense: 5'-GGCCAGACCAAAGATAGAGT-3'

Osteocalcin Sense: 5'-CTCACACTCCTCGCCCTATT-3' Antisense: 5'-GGTCAGCCAACTCGTCACA-3'

$\mathrm{Coll}$

Sense: 5'-GTGTAAGCGGTGGTGGTT-3'

Antisense: 5'-GGCCGGATACAGGTTT-3'

Osteonectin Sense: 5'-GATGAGGACAACAACCTTCTGAC-3' Antisense: 5'-TTAGATCACAAGATCCTTGTCGAT-3'

RANKL Sense: 5'-TGGATCACAGCACATCAGAGCAGAG-3' Antisense: 5'-ATACTCTGTAGCTAGGTCTCCTGAAG-3'

TRAIL

Sense: 5'-AAGCAGATGCAGGACAAGTACTCC-3' Antisense: 5'-CCCAGAGCCTTTTCATTCTTGGAG-3'

GAPDH Antisense: 5'-AAGTGGTCGTTGAGGGCAAT-3' 


\section{Table 3}

Real time RT-PCR analysis of gene expression in unsorted and sorted cells from three subjects

\begin{tabular}{lcccc} 
Cell population & \multicolumn{4}{c}{ Gene } \\
& AP & Col I & Osteocalcin & Osteonectin $^{\mathrm{A}}$ \\
Unsorted cells & $1.6(1.0)$ & $0.03(1.0)$ & $0.40(1.0)$ & $1.1(1.0)$ \\
BAP $^{+} /$RANKL $^{+}$ & $3.2(2.0)$ & $22.2(740)$ & $1.8(4.5)$ & $6.8(6.2)$ \\
BAP $^{+} /$RANKL $^{-}$ & $0.5(0.3)$ & $3.0(100)$ & $0.48(1.2)$ & $5.8(5.3)$ \\
${\text { BAP- } / \text { RANKL }^{+}}^{-}$ & ND & ND & ND & $1.5(1.4)$
\end{tabular}

Shown are the mean gene/GAPDH ratios for each gene. Numbers in parentheses indicate the relative abundance of each gene (normalized to GAPDH) as compared with unsorted marrow cells (arbitrarily assigned a value of 1.0 ). ARatio $\times 10^{4}$. ND, not detectable.

The mean fluorescence is obtained from the statistical analysis of the fluorescence height of the FITC emission and the mean value of the $x$ axis displayed by the software. This parameter is measured independently from the number of positive cells. This parameter measures the difference between the mean fluorescence of positive cells and that of the control sample, regardless of the frequency of positive cells. The primary probes were OPG-Fc-FITC (20) for sorting cells expressing RANKL and the anti-BAP, antiCD3, and anti-CD20 Ab's with PE-conjugated secondary Ab's for sorting MSCs, T lymphocytes, and B lymphocytes, respectively. The control for the OPGFc-FITC was a human Fc-FITC, and the controls for the primary Ab's for BAP, CD3, or CD20 were unrelated IgG isotypes. We used saturating amounts of OPG-Fc-FITC previously determined by doseresponse experiments (data not shown). A single lot of OPG-Fc-FITC was used for all studies.

Characterization of MSCs. In some experiments, we collected fractions of cells by FACS and studied them further for characteristics of the osteoblast phenotype. $\mathrm{BAP}^{+} / \mathrm{RANKL}^{+}$cells were plated at $5 \times 10^{4} /$ well in six-well plates and cultured for 21 days in a growth medium that maintains the cells, but does not differentiate them, or in a medium that allows them to differentiate to mature osteoblasts, as previously described (21). Briefly, the growth medium consisted of $\alpha$-MEM plus $10 \%$ FCS, whereas the differentiation medium consisted of the growth medium plus dexamethasone $\left(10^{-8} \mathrm{M}\right), \beta$-glycerol-phosphate $(10 \mathrm{mM})$, and L-ascorbate-phosphate $(100 \mu \mathrm{M})$. Mineralized nodules were assessed qualitatively by von Kossa staining and quantitatively by alizarin red-S staining followed by elution and colorimetric measurement (22). $\mathrm{BAP}^{+} / \mathrm{RANKL}^{-}$and $\mathrm{BAP}^{-} / \mathrm{RANKL}^{+}$cells did not grow under the same culture conditions.

In other samples of FACS-sorted cells, bone-related gene expression was assessed by real-time RT-PCR and by conventional RT-PCR. For these analyses, total RNA was purified from aliquots of the sorted cells using the StrataPrep Total RNA Miniprep Kit (Stratagene). Standard methods were used to prepare cDNA and for conventional PCR (23). Real-time PCR was performed using a Light Cycler (Roche Diagnostics $\mathrm{GmbH}$, Mannheim, Germany), as previously described (23). Table 2 shows the specific primers used for each of the genes analyzed.

Biochemical measurements. Serum C-terminal telopeptide of type I collagen (CTx) was measured using CrossLapsTM One Step ELISA (Osteometer Biotech A/S, Herlev, Denmark) (coefficient of variation [CV] $5.0 \%$ ). Urine $\mathrm{N}$-telopeptide of type I collagen (NTx) was determined by a competitive immunoassay (Osteomark; Ostex International Inc., Seattle, Washington, USA) (CV 6.3\%), and results were expressed as the $\mathrm{NTx} /$ creatinine ratio. Serum $17 \beta$-estradiol was measured by a high-sensitivity double $\mathrm{Ab}$ radioimmunoassay with a detection limit of $5 \mathrm{pg} / \mathrm{ml}$ (Diagnostic Products Corp., Los Angeles, California, USA) (CV 3.8\%). Serum OPG was determined by ELISA (24) (CV <15\%) and serum RANKL by an ELISA kit (BioNet Inc., Southbridge, Massachusetts, USA) (CV 6\%).

Statistical analyses. Differences among groups were performed by one-way ANOVA followed by the StudentNewman-Keuls test, which adjusts for multiple comparisons. Data are presented as mean plus or minus SEM.

\section{Results}

Clinical characteristics and biochemical measurements

Baseline values for the three groups of women are given in Table 1. The clinical characteristics were similar in all groups. As expected, the estrogen-deficient women (Group B) had lower values for serum estradiol and higher values for bone resorption markers (serum CTx
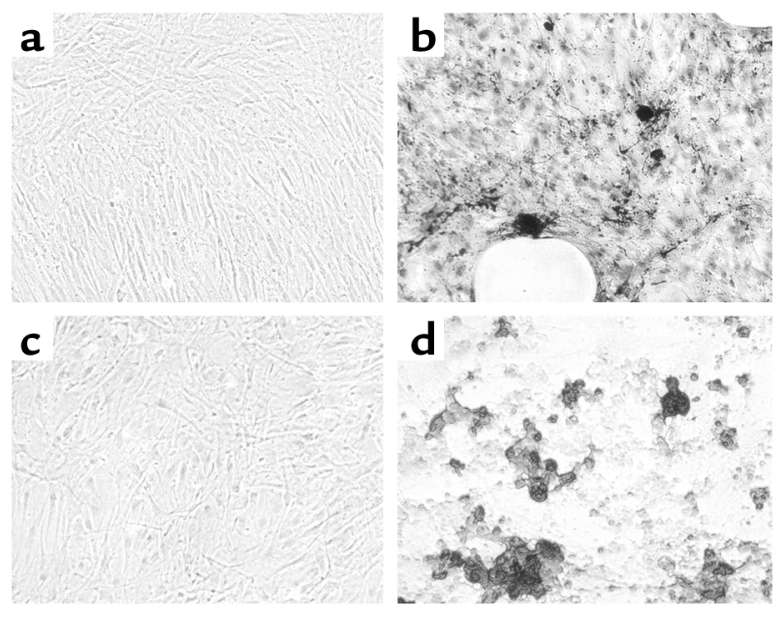

\section{Figure 2}

Formation of mineralized nodules during culture. $\mathrm{BAP}^{+} / \mathrm{RANKL}^{+}$ cells were cultured in either nutrient medium ( $\mathbf{a}$ and $\mathbf{c}$ ) or in osteoblast differentiation medium (b and $\mathbf{d}$ ) for 21 days. When stained using von Kossa stain ( $\mathbf{a}$ and $\mathbf{b}$ ) or with alizarin red-S ( $\mathbf{c}$ and d), mineralized nodules were apparent when cells were cultured in osteoblast differentiation medium. The $\mathrm{BAP}^{+} / \mathrm{RANKL}^{-}$and BAP-/RANKL ${ }^{+}$cells did not proliferate sufficiently in culture to allow mineralization to be assessed. 
Table 4

Percentages of bone marrow mononuclear cells expressing various surface markers as assessed by flow cytometry

\begin{tabular}{|c|c|c|c|c|c|c|c|}
\hline Cell markers (cell type) & $\begin{array}{c}\text { Group A } \\
n=12\end{array}$ & $\begin{array}{c}\text { Group B } \\
n=12\end{array}$ & $\begin{array}{c}\text { Group C } \\
n=12\end{array}$ & $P^{A}$ & A vs. $B^{B}$ & A vs. $C^{B}$ & B vs. $C^{B}$ \\
\hline \multicolumn{8}{|l|}{ Single-color } \\
\hline $\mathrm{BAP}^{+}$ & $9.7 \pm 1.2$ & $3.4 \pm 0.4$ & $6.8 \pm 1.0$ & $<0.01$ & $<0.05$ & NS & $<0.05$ \\
\hline $\mathrm{CD}^{+}(\mathrm{T}$ cells $)$ & $33.2 \pm 2$ & $40.8 \pm 2.6$ & $37.2 \pm 2.5$ & NS & & & \\
\hline $\mathrm{CD}^{20^{+}}$(B cells $)$ & $4.4 \pm 0.4$ & $6.3 \pm 0.7$ & $6.4 \pm 0.7$ & NS & & & \\
\hline $\mathrm{RANKL}^{+}$ & $5.7 \pm 0.5$ & $6.4 \pm 0.7$ & $5.7 \pm 0.6$ & NS & & & \\
\hline \multicolumn{8}{|l|}{ Dual-color } \\
\hline $\mathrm{BAP}^{+} / \mathrm{RANKL}^{+}(\mathrm{MSCs})$ & $2.3 \pm 0.2$ & $1.1 \pm 0.1$ & $1.6 \pm 0.1$ & $<0.001$ & $<0.05$ & NS & $<0.05$ \\
\hline $\mathrm{CD}^{+} / \mathrm{RANKL}^{+}$ & $1.0 \pm 0.1$ & $1.1 \pm 0.2$ & $1.7 \pm 0.4$ & NS & & & \\
\hline $\mathrm{CD} 20^{+} / \mathrm{RANKL}^{+}$ & $0.8 \pm 0.1$ & $0.5 \pm 0.1$ & $0.6 \pm 0.5$ & NS & & & \\
\hline
\end{tabular}

and urine NTx) than did the estrogen-replete women (Groups A and C), and these two groups did not differ from each other. Serum levels of OPG and RANKL did not differ among groups.

\section{Validation of flow-cytometry method}

Characterization of the MSCs. After passage of bone marrow cells through a Ficoll-Paque density gradient to obtain mononuclear cells, we employed dual-color flow cytometry to obtain $\mathrm{BAP}^{+} / \mathrm{RANKL}^{+}$cells (Figure 1 ). To establish that these were authentic MSCs of the osteoblast lineage, we made further studies. First, using dual-color flow cytometry, we showed that an average of $27 \%$ of cells expressing BAP coexpressed RANKL (derived from Table 4), a characteristic finding in osteoblastic lineage cells (25). Second, from a mean of four flow-cytometry analyses, we found that $63 \%$ of the $\mathrm{BAP}^{+}$cells coexpressed STRO-1 (data not shown), a well-characterized surface marker for early osteoblast precursor cells $(26,27)$. Third, when expression of osteoblast-related genes was measured by real time RT-PCR, we found a marked enrichment of these genes in the $\mathrm{BAP}^{+} / \mathrm{RANKL}^{+}$cells as compared with the unsorted cells or the $\mathrm{BAP}^{+} / \mathrm{RANKL}^{-}$or $\mathrm{BAP}^{-} / \mathrm{RANKL}^{+}$cells (Table 3 ). The only exception to this was AP gene expression, which was increased in the $\mathrm{BAP}^{+} / \mathrm{RANKL}^{+}$cells approximately twofold as compared with unsorted cells only. This relatively small enrichment for AP is likely due to the presence of $\mathrm{AP}^{+}$granulocyte precursors in the Ficoll-Paque passaged but unsorted bone marrow cells. These cells were excluded from the sorted $\mathrm{BAP}^{+} / \mathrm{RANKL}^{+}$cells by gating. $\mathrm{BAP}^{+} / \mathrm{RANKL}^{-}$cells expressed much lower levels of the osteoblast-specific genes, whereas these genes were largely undetectable in the $\mathrm{BAP}^{-} / \mathrm{RANKL}^{+}$ cells, with the exception of osteonectin, which was present at low levels (Table 3). Finally, sorted $\mathrm{BAP}^{+} / \mathrm{RANKL}^{+}$cells were cultured for 21 days in an osteoblast differentiation medium. As previously described (25), the $\mathrm{BAP}^{+} / \mathrm{RANKL}^{+}$cells that were maintained in a nondifferentiating medium failed to develop mineralized nodules, whereas those grown in osteoblast differentiation medium formed mineralized nodules that stained positively for calcium by the von Kossa stain or alizarin red-S (Figure 2). When dye was eluted from nodules stained with alizarin red-S and measured quantitatively, $\mathrm{BAP}^{+} / \mathrm{RANKL}^{+}$ cells grown in osteoblast differentiation medium for 21 days had a 20 -fold increase in dye as compared with control $\mathrm{BAP}^{+} / \mathrm{RANKL}^{+}$cells maintained in nutrient medium.

Exclusion of granulocytes. Because precursor cells of the granulocyte lineage are present in bone marrow cells after Ficoll-Paque passage, and these also express AP, studies were made to ensure that these did not contaminate the $\mathrm{BAP}^{+} / \mathrm{RANKL}^{+}$sorted cells.

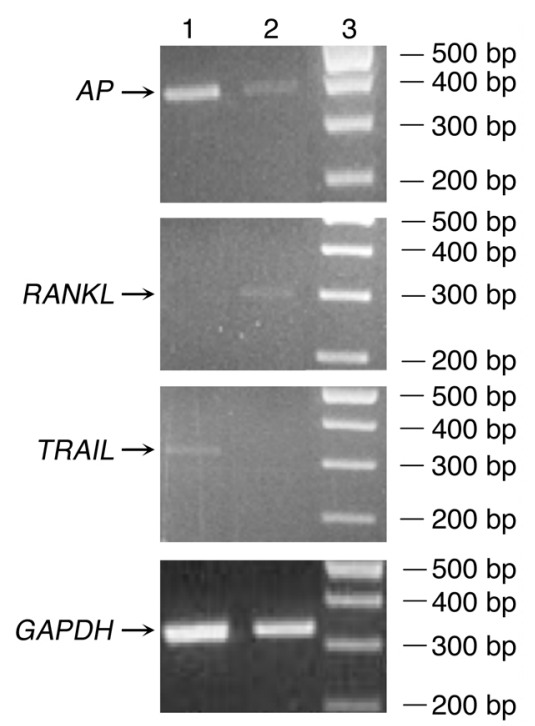

Figure 3

Demonstration of the lack of TRAIL expression in cells sorted by FACS using RT-PCR analysis. In ethidium bromide-stained agarose gel of PCR products, $\mathrm{BAP}^{+} / \mathrm{RANKL}^{-}$cells sorted by dual-color flow cytometry are shown in lane 1 and $\mathrm{BAP}^{+} / \mathrm{RANKL}^{+}$cells are shown in lane 2 . Size markers are given in lane 3 . Note that the $\mathrm{BAP}^{+}$cells binding the OPG-Fc-FITC probe (lane 2) express the RANKL, but not TRAIL mRNA. Conversely, the $\mathrm{BAP}^{+}$cells not binding the OPG-Fc-FITC probe (lane 1) express TRAIL mRNA, but do not express RANKL. 


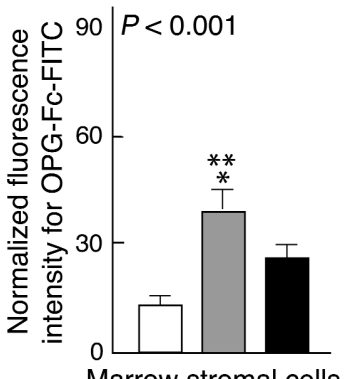

Marrow stromal cells

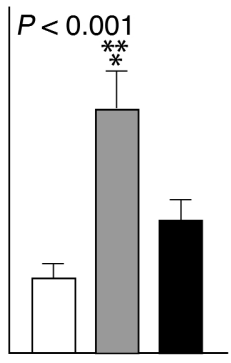

B cells

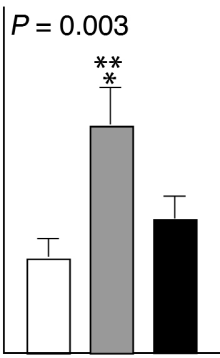

T cells

\section{Figure 4}

Changes in OPG-Fc-FITC fluorescence as an index of mean RANKL surface concentration per cell (mean \pm SEM). The premenopausal women (Group A) are shown by the white bars, the untreated postmenopausal women (Group B) by gray bars, and the estrogen-treated postmenopausal women (Group C) by black bars. There is a highly significant (ANOVA $P$ values as indicated) difference among three groups. There is a two- to threefold significant increase of the mean RANKL fluorescence intensity per cell in Group B over Group A $\left({ }^{*}\right)$ and a somewhat smaller significant increase in Group B over Group $C\left({ }^{*}\right)$ as analyzed by the Student-Newman-Keuls test.

As reported previously (28), the population of granulocyte precursors was recognized by dot plot clustering in the $\mathrm{R} 2$ region based on forward/side scatter nonfluorescent signals. Granulocyte precursors were then excluded by gating on $\mathrm{R} 1$ region and excluding the $\mathrm{R} 2$ region (Figure 1a). The effectiveness of this exclusion was demonstrated by using an $\mathrm{Ab}$ directed against the CD15 surface marker that is characteristic for cells of the granulocyte lineage. When sorted by dual-color flow cytometry and gated on R1 on lymphocyte/monocyte populations, $\mathrm{BAP}^{+} / \mathrm{CD} 15^{+}$ coexpression was below the level of background fluorescence of the control isotype (data not shown). Similarly, when two-color flow cytometry with OPGFc-FITC probe and anti-CD15 Ab was employed, gated on R1 and excluding R2, the RANKL ${ }^{+} / \mathrm{CD} 15^{+}$ coexpression was also below the background level. Furthermore, in three-color flow cytometry with anti-BAP Ab, OPG-Fc-FITC probe, and anti-CD15 $\mathrm{Ab}, \mathrm{BAP}^{+} / \mathrm{RANKL}^{+}$cells did not coexpress the granulocyte marker CD15 above the control background activity (data not shown).

Assessment of effect of TRAIL. In certain experimental conditions, OPG may bind to TRAIL (29), whereas in other experimental conditions, it does not $(20,30)$. Thus, studies were made to determine if binding of OPG-Fc-FITC to TRAIL could have affected our results. In $\mathrm{BAP}^{+} / \mathrm{RANKL}^{-}$cells sorted by dual-color flow cytometry, we used conventional RT-PCR to demonstrate that TRAIL mRNA was expressed but that RANKL mRNA was not (Figure 3). In contrast, in $\mathrm{BAP}^{+} / \mathrm{RANKL}^{+}$sorted cells, RANKL mRNA, but not TRAIL mRNA, was expressed. Moreover, when cells were sorted
Table 5

Correlation coefficients between mean fluorescence intensity per cell for OPG-Fc-FITC, an index of the surface concentration of RANKL by flow cytometry, and biochemical variables of all subjects in merged groups

\begin{tabular}{lccc}
\hline Cell markers (cell type) & Serum CTx & Urine NTx & Serum $\mathrm{E}_{2}$ \\
$\mathrm{BAP}^{+} / \mathrm{RANKL}^{+}(\mathrm{MSCs})$ & $0.48(P=0.002)$ & $0.42(P=0.009)$ & $-0.30(P=0.07)$ \\
$\mathrm{CD}^{+} / \mathrm{RANKL}^{+}(\mathrm{T}$ cells $)$ & $0.36(P=0.02)$ & $0.34(P=0.03)$ & $-0.31(P=0.06)$ \\
${\mathrm{CD} 20^{+} / \mathrm{RANKL}^{+}(\mathrm{B} \text { cells })}^{0.49(P=0.002)}$ & $0.49(P=0.002)$ & $-0.31(P=0.06)$ \\
Total RANKL ${ }^{+}$cells & $0.37(P=0.02)$ & $0.44(P=0.007)$ & $-0.26(P=0.01)$ \\
\hline
\end{tabular}

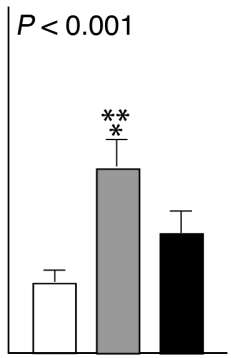

Total RANKL cells by single-color flow cytometry, preincubation with an anti-TRAIL $\mathrm{Ab}$ did not affect the proportion of cells binding to OPG-Fc-FITC, and preincubation with OPG-Fc did not affect the proportion of cells binding to an anti-TRAIL Ab (data not shown). Thus, under the conditions of our study, TRAIL does not appear to affect our experimental results.

Flow cytometry analysis of cell types coexpressing RANKL

The frequency of cell types based on flow-cytometry analysis was reported as the percentage of the gated cells in R1. Table 4 summarizes the proportion of cells gated from R1 that were sorted with anti-BAP (MSCs), anti-CD3 ( $\mathrm{T}$ cells), anti-CD20 (B cells) Ab's, and OPG-Fc-FITC (total RANKL-expressing cells) by single-color flow cytometry and the MSCs, T cells, and B cells that coexpressed RANKL sorted by dual-color flow cytometry. For MSCs, the proportion of both those cells expressing $\mathrm{BAP}\left(\mathrm{BAP}^{+}\right.$cells) and those coexpressing RANKL $\left(\mathrm{BAP}^{+} / \mathrm{RANKL}^{+}\right)$cells were decreased by more than half in Group B as compared with Group A and were decreased somewhat less when compared with Group C (ANOVA, $P<0.01$ and $P<0.001$, respectively). The proportion of $\mathrm{T}$ cells, $\mathrm{B}$ cells, and total RANKL $^{+}$cells by single-color flow cytometry or $\mathrm{T}$ cells or B cells coexpressing RANKL by dual-color flow cytometry was not different among groups. From the merged data of Groups A, B, and C in Table 4, we obtained the fraction of each cell type expressing RANKL by dividing the average value in the three groups for dual color sorting (proportion expressing both the cell type marker and the surface marker RANKL) by the average value for single-color sorting (proportion of gated bone marrow mononuclear cells with the characteristic cell type marker). We found that $1 / 4$ of MSCs, $1 / 9$ of B cells, but only $1 / 29$ of $T$ cells coexpressed RANKL. A representative dot plot analysis for single- and dual color-sorted cells is shown in Figure 1. 


\section{Relative intensity of OPG-Fc-FITC fluorescence}

We estimated the surface concentration of RANKL per cell by measuring the average OPG-Fc-FITC fluorescence intensity per cell for RANKL-expressing MSCs, T cells, B cells, and for total RANKL-expressing cells in R1 (Figure 4). As compared with Group A (premenopausal women), the average fluorescence intensity was increased in Group B (untreated postmenopausal) by $322 \%(P<0.001)$ in the MCSs, by $260 \%$ $(P<0.01)$ in the T cells, by $238 \%(P<0.001)$ in the B cells, and by $293 \%(P<0.01)$ for total $\mathrm{RANKL}^{+}$gated cells. Findings were similar when Group B was compared with Group C, and there were no significant differences between Groups A and C.

Table 5 gives the correlation coefficients for the relative intensity of OPG-Fc-FITC fluorescence, an index of surface RANKL concentration per cell, versus the concentration of bone resorption markers (serum CTx and urine NTx) and serum estradiol concentration. RANKL fluorescence was positively and significantly correlated with levels of bone resorption markers for all three cell types coexpressing RANKL and for total RANKL-expressing cells. Moreover, there was a significant negative correlation with serum estradiol levels with total RANKL-expressing cells. For RANKLexpressing MSCs, B cells, and T cells, this correlation was also negative, but of borderline significance. Serum levels of RANKL, OPG, or the RANKL/OPG ratio did not correlate with OPG-Fc-FITC fluorescence or with the proportion of MSCs, T cells, B cells, or the total RANKL-expressing cells (data not shown).

\section{Discussion}

Almost all studies assessing the paracrine mediation of estrogen action have been made in rodents or use human bone cell lines or primary cultures of cells from orthopedic samples in vitro. Thus far, estrogenregulation of bone marrow cytokines has not been studied directly in humans despite evidence that estrogen action on bone may differ substantially in humans and mice. For example, ER $\alpha$ knockout mice have short limbs and mild osteopenia (31), whereas a young adult male with homozygous null mutations of ER $\alpha$ had elongated limbs and a greater degree of osteopenia (32). Another, and possibly more relevant, example is that IL- 6 consistently upregulates RANKL in murine cells in vitro (33), but fails to do so in human cells (34).

Our major objective was to determine whether expression of RANKL by bone marrow cells contributes to the increased bone resorption in early postmenopausal women. Thus, in estrogen-deficient and estrogen-replete women, we obtained bone marrow mononuclear cells, used dual-color flow cytometry to identify cells expressing RANKL, and characterized them as MSCs, T cells, or B cells, using Ab's for bone alkaline phosphatase, CD3, and CD20, respectively. We then assessed the proportion of these cells expressing RANKL and the relative fluorescence intensity of OPG-Fc-FITC as an index of the surface concentration of RANKL per cell.

Isolation of T cells and B cells by single-color flow cytometry is a well-accepted, routine procedure, whereas flow cytometry has not been heretofore employed to isolate MSCs. Previous investigators have employed plastic adherence $(35,36)$ or the STRO-1 Ab (26) to isolate MSCs. The plastic adherence method selects only relatively small number of MSCs and, because these cells must be expanded in culture, it is not useful for assessing RANKL expression in vivo. Although the STRO- 1 Ab recognizes a subset of early MSCs, 95\% of the detected cells are erythroid precursors that must be excluded by using an $\mathrm{Ab}$ against glycophorin $\mathrm{A}$, thus complicating the flow-cytometry procedure. Moreover, the role of the STRO-1 epitope in osteoblast function is unknown. For these reasons, we elected to isolate MSCs by flow cytometry using an $\mathrm{Ab}$ directed against BAP, an ectoenzyme that plays an important role in osteoblast function. Although granulocyte precursors in bone marrow also express this AP isoenzyme, we were able to identify and exclude contamination by this cell type by its light scatter characteristics during flow cytometry. Moreover, using an Ab for CD15, a specific granulocyte marker (37), we confirmed that the electronic gate used to sort MSCs did not contain granulocyte lineage cells.

Cells coexpressing BAP and RANKL represent about $1-3 \%$ of the bone marrow mononuclear cells contained in the R1 gate. We demonstrated that these were authentic MSCs by several criteria. First, about one-fourth of the $\mathrm{BAP}^{+}$cells coexpressed RANKL, a characteristic of MSCs (25). Indeed, other than osteoblast lineage cells, no other cell type is known to coexpress both surface markers. Second, half of the $\mathrm{BAP}^{+}$cells coexpressed the STRO-1 epitope, an established surface marker for early osteoblast progenitor cells $(26,27)$. Third, when isolated by FACS, $\mathrm{BAP}^{+} / \mathrm{RANKL}^{+}$cells were shown to express high concentrations of representative bonerelated genes - BAP, Col 1, osteocalcin, and osteonectin - by real-time RT-PCR. Finally, and most importantly, $\mathrm{BAP}^{+} / \mathrm{RANKL}^{+}$cells were able to form multiple mineralized nodules, the sine qua non for osteoblast lineage cells, after 21 days of culture in an osteoblast differentiation medium.

We also assessed nonspecific binding of the OPGFc-FITC probe. One potential confounder was binding of the OPG-Fc-FITC to TRAIL, a related TNF family member with potent apoptosis-inducing properties that is expressed on surfaces of many cell types $(38,39)$. OPG has been reported both to bind to TRAIL (29) and not to bind to it $(20,30)$, depending on the experimental conditions. Mice in whom the TRAIL gene was deleted, however, had normal bone rather than the osteopetrotic bone found in transgenic mice overexpressing OPG (40). This suggests 
that TRAIL was not capable of substantially neutralizing OPG in vivo. For several reasons, we believe that binding of the OPG-Fc-FITC probe to TRAIL is not relevant to our results. First, the $\mathrm{BAP}^{+} / \mathrm{RANKL}^{+}$cells that bind OPG-Fc-FITC did not express TRAIL mRNA as assessed by RT-PCR. Second, OPG-Fc-FITC binding to the $\mathrm{BAP}^{+} / \mathrm{RANKL}^{+}$cells was not affected by preincubation with TRAIL Ab, as assessed by either single- or dual-color flow cytometry. Moreover, preincubation with saturating concentrations of OPG-Fc did not affect the percentage of $\mathrm{TRAIL}^{+}$cells assessed by single-color flow cytometry. Finally, and importantly, since TRAIL does not stimulate osteoclastogenesis (29), its expression would not be expected to correlate with bone resorption markers. Yet, we found that OPG-Fc-FITC fluorescence intensity was strongly correlated to bone resorption markers. Another potential confounder was nonspecific binding of the Fc component of the OPG-Fc-FITC probe to Fc receptors. This was controlled by blocking Fc receptors in the cell samples with an excess of normal serum. To assess residual nonspecific binding, we used the $\mathrm{Fc}$ fragment of human IgG conjugated to FITC as a control, and we accepted the unspecific (background) activity of the control at a level that was less than $5 \%$ of the specific activity of OPG-Fc-FITC. Finally, other nonspecific binding of full-length OPG is mainly to heparin, and this was eliminated by use of a N-terminal OPG-Fc fusion probe in which the heparin-binding domain was deleted (20).

The effect of menopause on RANKL regulation of bone resorption was studied by comparing results in estrogen-deficient, early postmenopausal women (Group B) with two estrogen-replete control groups - premenopausal women (Group A) and age-comparable postmenopausal women who were receiving transdermal estradiol patches (Group C). We found no difference between Groups $\mathrm{A}$ and $\mathrm{C}$ in any of the experimental variables, eliminating the possibilities that changes in the untreated postmenopausal women (Group B) were due to age differences between Groups A and B or to differences between ovarian estrogen production and transdermal estrogen replacement in Group C.

Using single-color flow cytometry, we found no significant differences for the proportions of $\mathrm{T}$ cells or B cells among the three clinical groups of women, either for the total cells or for those that coexpressed RANKL. This contrasts with findings in mice where ovariectomy increases bone marrow $\mathrm{T}$ cells twofold (6) and B lymphocytes twofold $(14,41)$, changes that have been suggested to be the cause of bone loss in estrogen deficiency. This striking species disparity reemphasizes the need for human studies to confirm studies of bone biology that have been made in rodents. We cannot exclude the possibility, however, that the total number of bone marrow mononuclear cells were increased but the proportion of $\mathrm{T}$ cells and $B$ cells did not change.
In contrast to the lack of change in the proportions of $T$ cells and $B$ cells, we found that the estrogen-deficient group of women (Group B) had only about half of the proportion of MSCs that estrogen-sufficient women (Groups A and C) did. This was unexpected, because many studies have shown that estrogen deficiency increases the numbers of mature osteoblasts on endosteal surfaces of bone $(1,3)$. These results, however, are consistent with a rapid-throughput kinetic model in which a compartment of preosteoblastic cells is reduced in the setting of estrogen deficiency by the increased demand for osteoblasts on bone surfaces. They are also consistent with a model in which mature osteoblasts on bone surfaces originate at least partially from other sources, such as lining cells (42) or vascular endothelial cells (43). Gregorio et al. (44) found that ovariectomy in rats increased the number of early osteoblastic colonyforming units in bone marrow. Their findings contrast with the decrease in the compartment of $\mathrm{BAP}^{+} / \mathrm{RANKL}^{+}$osteoblastic cells that we identified. This implies the $\mathrm{BAP}^{+} / \mathrm{RANKL}^{+}$cells that we sorted may represent an intermediate stage of differentiating osteoblast lineage cells. Alternatively, the discrepancy between the study of Gregorio et al. and ours could indicate another species difference between rodents and humans.

In the gated bone marrow mononuclear cells, there were threefold more $\mathrm{T}$ cells than $\mathrm{BAP}^{+}$cells, whereas the number of $\mathrm{B}$ cells was about half that of the $\mathrm{BAP}^{+}$. Only 1 in 29 of the T cells, however, coexpressed RANKL, whereas 1 in 4 of the $\mathrm{BAP}^{+}$cells and 1 in 9 of the $\mathrm{B}$ cells did. Thus, MSCs represented 40\% (1:2.4) of the total RANKL-expressing cell types in the estrogen-replete groups, but only $17 \%$ (or 1:6) in the estrogen-deficient postmenopausal women. Nonetheless, because their estimated surface concentration of RANKL increased in the untreated postmenopausal women more than their numbers decreased, the MSCs appear to contribute as much to total RANKL activity as did the T cells and B cells, even in the untreated postmenopausal women.

Nonetheless, T cells appear to make a substantial contribution to RANKL activity in bone marrow. The expression of RANKL by T cells may be related, in part, to their function as immunoregulators, because targeted disruption of the RANKL gene results in mice lacking lymph nodes and with defective $T$ and $B$ lymphocyte differentiation (45). There also is strong evidence, however, that RANKL expression by $\mathrm{T}$ cells upregulates osteoclastogenesis and thus may contribute to bone loss when it is increased. In an experimental mouse model of inflammatory arthritis, activated $\mathrm{T}$ lymphocytes expressing RANKL were shown to regulate the associated periarticular bone loss and joint destruction (46). Also, $\mathrm{T}$ cell-deficient mice have impaired osteoclastogenesis and do not lose bone after ovariectomy (6).

The most important finding of our study was a two- to threefold increase in relative fluorescence intensity of OPG-Fc-FITC, an index of the surface 
concentration of RANKL molecules, in the estrogendeficient postmenopausal women. This increase occurred in all three RANKL-expressing bone marrow cell types and in total RANKL-expressing cells. Although the proportion of gated RANKL ${ }^{+}$MSCs decreased by about half in the untreated postmenopausal women as compared with the premenopausal women, the apparent surface concentration of RANKL on MSCs increased more than threefold. The proportion of gated $\mathrm{RANKL}^{+} \mathrm{T}$ and $\mathrm{B}$ cells was increased slightly after menopause, and their apparent surface concentration of RANKL also increased. Thus, overall bone marrow availability of RANKL was increased.

Our findings that OPG-Fc-FITC fluorescence correlated directly with bone resorption markers in all three types of RANKL-expressing cell types and was negatively correlated with serum $17 \beta$-estradiol levels for total marrow RANKL-expressing cells suggest a causal relationship. This relationship is likely to be physiologic because the cells were obtained from the bone marrow microenvironment where they were in equilibrium with the prevailing cytokine milieu and studied ex vivo soon thereafter. This does not necessarily establish, however, that estrogen regulates RANKL directly. In vitro studies have shown that IL- $1 \alpha$, TNF- $\alpha$, and $\mathrm{PGE}_{2}$ upregulate $\operatorname{RANKL}(10,11)$, and these cytokines have been reported to be increased by estrogen deficiency $(1,3,4)$. Thus, we cannot exclude the possibility that estrogen acts indirectly by stimulating these or other estrogen-dependent cytokines to upregulate RANKL.

Despite clear changes in RANKL expression on marrow cells, we did not detect differences in circulating RANKL levels in the three groups, highlighting the importance of assessing levels of these factors directly in the bone microenvironment. Moreover, although we and others have demonstrated previously that estrogen stimulates OPG production both in vitro $(15,16)$ and in vivo $(17)$, circulating OPG levels did not differ between the three groups. We believe that this may occur because estrogen deficiency and increased bone turnover affect OPG production in opposite directions with estrogen deficiency decreasing and increased bone turnover increasing OPG production $(24,47)$. These opposing effects may cancel each other, resulting in no net change in OPG levels in postmenopausal women who have both chronic estrogen deficiency and increased bone turnover. In our previous study of experimentally induced acute hypogonadism in men, however, we were able to dissociate these two effects experimentally and, consistent with the in vitro data, demonstrate that estrogen has a stimulatory effect on OPG levels (17).

In conclusion, we have developed a new method for sorting bone marrow cells expressing RANKL and for estimating its surface concentration. With this method, we show that RANKL-expression of MSCs,
$T$ cells, and $B$ cells is upregulated two- to threefold by estrogen deficiency and correlated directly with increases in bone resorption markers and inversely with serum $17 \beta$-estradiol for total RANKL-expressing cells. The mechanism of estrogen suppression of osteoclast formation and function is complex and involves multiple cytokine mediators $(1,3,4)$. Because RANKL, however, is a highly potent, final effector of osteoclast formation and function, our demonstration that it is increased by estrogen deficiency and decreased by estrogen sufficiency in three bone marrow cell types suggests that it may play a major role in mediating increased bone resorption and bone loss following menopause.

\section{Acknowledgments}

We wish to thank Kelly Hoey for performing the biochemistry assays, James M. Peterson for assistance with data management, and Paul J. Leibson and James E. Tarara for helpful advice on flow-cytometry procedures and analysis. This work was supported in part by grants P01 AG-04875 and M01 RR00585 from the NIH, the U.S. Public Health Service, and from Amgen Inc.

1. Riggs, B.L., Khosla, S., and Melton, L.J. 2002. Sex steroids and the construction and conservation of the adult skeleton. Endocr. Rev. 23:279-302.

2. Khosla, S., Melton, L.J., and Riggs, B.L. 2002. Estrogen and the male skeleton. J. Clin. Endocrinol. Metab. 87:1443-1450.

3. Manolagas, S.C. 2000. Birth and death of bone cells: basic regulatory mechanisms and implications for the pathogenesis and treatment of osteoporosis. Endocr. Rev. 21:115-137.

4. Pacifici, R. 1996. Estrogen, cytokines, and pathogenesis of postmenopausal osteoporosis. J. Bone Miner. Res. 11:1043-1051.

5. Tanaka, S., et al. 1993. Macrophage colony-stimulating factor is indispensable for both proliferation and differentiation of osteoclast progenitors. J. Clin. Invest. 91:257-263.

6. Cenci, S., et al. 2000. Estrogen-deficiency induces bone loss by enhancing T-cell production of TNF- $\alpha$. J. Clin. Invest. 106:1229-1327.

7. Lam, J., et al. 2000. TNF- $\alpha$ induces osteoclastogenesis by direct stimulation of macrophages exposed to permissive levels of RANK ligand. J. Clin. Invest. 106:1481-1488.

8. Oursler, M.J., et al. 1991. Modulation of transforming growth factor- $\beta$ production in normal human osteoblast-like cells by $17 \beta$-estradiol and parathyroid hormone. Endocrinology. 129:3313-3320.

9. Hughes, D.E., et al. 1999. Estrogen promotes apoptosis of murine osteoclasts mediated by TGF- $\beta$. Nat. Med. 2:1132-1136.

10. Suda, T., et al. 1999. Modulation of osteoclast differentiation and function by the new members of the tumor necrosis factor receptor and ligand families. Endocr. Rev. 20:345-357.

11. Hofbauer, L.C., et al. 2000. The roles of osteoprotegerin and osteoprotegerin ligand in the paracrine regulation of bone resorption. J. Bone Miner. Res. 15:2-12.

12. Khosla, S. 2001. Minireview: The OPG/RANKL/RANK system. Endocrinology. 142:5050-5055.

13. Yasuda, H., et al. 1998. Osteoclast differentiation factor is a ligand for osteoprotegerin/osteoclastogenesis-inhibitory factor and is identical to TRANCE/RANKL. Proc. Natl. Acad. Sci. U. S. A. 95:3597-3602.

14. Kanematsu, M., et al. 2000. Prostaglandin E2 induces expression of receptor activator of nuclear factor-kB ligand/osteoprotegrin ligand on pre-B cells: implications for accelerated osteoclastogenesis in estrogen deficiency. J. Bone Miner. Res. 15:1321-1329.

15. Hofbauer, L.C., et al. 1999. Estrogen stimulates gene expression and protein production of osteoprotegerin in human osteoblastic cells. Endocrinology. 140:4367-4370.

16. Saika, M., Inoue, D., Kido, S., and Matsumoto, T. 2001. 17 $\beta$-Estradiol stimulates expression of osteoprotegerin by a mouse stromal cell line, ST-2, via estrogen receptor- $\alpha$. Endocrinology. 142:2205-2212.

17. Khosla, S., Atkinson, E.J., Dunstan, C.R., and O'Fallon, W.M. 2002. Effect of estrogen versus testosterone on circulating osteoprotegerin and other cytokine levels in normal elderly men. J. Clin. Endocrinol. Metab. 87:1550-1554.

18. Shevde, N.K., Bendixen, A.C., Dienger, K.M., and Pike,J.W. 2000. Estrogens 
suppress RANK ligand-induced osteoclast differentiation via a stromal cell independent mechanism c-Jun repression. Proc. Natl. Acad. Sci. U. S. A 97:7829-7834.

19. Simonet, W.S., et al. 1997. Osteoprotegerin: a novel secreted protein involved in the regulation of bone density. Cell. 89:309-319.

20. Lacey, D.L., et al. 1998. Osteoprotegerin ligand is a cytokine that regulates osteoclast differentiation and activation. Cell. 93:165-176.

21. Gori, F., Thomas, T., Hicok, K.C., Spelsberg, T.C., and Riggs, B.L. 1999 Differentiation of human marrow stromal precursor cells: bone morphogenetic protein-2 increases OSF2/CBFA1, enhances osteoblast commitment, and inhibits late adipocyte maturation. J. Bone Miner. Res. 14:1522-1535.

22. Bodine, P.V.N., Trailsmith, M., and Komm, B.S. 1996. Development and characterization of a conditionally transformed adult human osteoblastic cell line. J. Bone Miner. Res. 11:806-819.

23. Locklin, R.M., et al. 2001. Assessment of gene regulation by bone morphogenetic protein 2 in human marrow stromal cells using gene array technology. J. Bone Miner. Res. 16:2192-2204.

24. Khosla, S., et al. 2002. Correlates of osteoprotegerin levels in women and men. Osteoporos. Int. 13:394-399.

25. Gori, F., et al. 2000. The expression of osteoprotegerin and RANK ligand and the support of osteoclast formation by stromal-osteoblast lineage cells is developmentally regulated. Endocrinology. 141:4768-4776.

26. Gronthos, S., Graves, S.E., Ohta, S., and Simmons, P.J. 1994. The STRO-1+ fraction of adult human bone marrow contains the osteogenic precursors. Blood. 84:4164-4173.

27. Stewart, K., et al. 1999. Further characterization of cells expressing STRO-1 in cultures of adult human bone marrow stromal cells. J. Bone Miner. Res. 14:1345-1356.

28. Naeim, F. 1998. Pathology of bone marrow. 2nd edition. Williams \& Wilkins. Baltimore, Maryland, USA. 61 pp.

29. Emery, J.G., et al. 1998. Osteoprotegerin is a receptor for the cytotoxic ligand TRAIL. J. Biol. Chem. 273:14363-14367.

30. Truneh, A., et al. 2000. Temperature-sensitive differential affinity of TRAIL for its receptors. DR5 is the highest affinity receptor. J. Biol. Chem. 275:23319-23325.

31. Couse, J.F., and Korach, K.S. 1999. Estrogen receptor null mice: what have we learned and where will they lead us. Endocr. Rev. 20:358-417.

32. Smith, E.P., et al. 1994. Estrogen resistance caused by a mutation in the estrogen-receptor gene in a man. N. Engl. J. Med. 331:1056-1061.

33. O'Brien, C.A., Gubrij, I., Lin, S.C., Saylors, R.L., and Manolagas, S.C.
1999. STAT3 activation in stromal/osteoblastic cells is required for induction of the receptor activator of NF-KB ligand and stimulation of osteoclastogenesis by gp130-utilizing cytokines or interleukin-1 but not 1,25-dihydroxyvitamin $\mathrm{D}_{3}$ or parathyroid hormone. J. Biol. Chem. 274:19301-19308.

34. Hofbauer, L.C., et al. 1999. Interleukin- $1 \beta$ and tumor necrosis factor- $\alpha$, but not interleukin-6, stimulate osteoprotegerin ligand gene expression in human osteoblastic cells. Bone. 25:255-259.

35. Owen, M., and Friedenstein, A.J. 1988. Stromal stem cells: marrowderived osteogenic precursors. Ciba Found. Symp. 136:42-60.

36. Kassem, M., Risteli, L., Mosekilde, L., Melsen, F., and Eriksen, E.F. 1991. Formation of osteoblast-like cells from human mononuclear bone marrow cultures. APMIS. 99:269-274.

37. Knapp, W. 1989. Leucocytes typing IV: white cell differentiation antigens. Oxford University Press. New York, New York, USA. 1182 pp.

38. Walczak, H., and Krammer, P.H. 2000. The CD95 (APO-1/Fas) and the TRAIL (APO-2L) apoptosis systems. Exp. Cell Res. 256:58-66.

39. Atkins, G.J., et al. 2000. Human osteoblasts are resistant to Apo2L/TRAIL-mediated apoptosis. Bone. 31:448-456.

40. Sedger, L.M., et al. 2002. Characterization of the in vivo function of TNF- $\alpha$-related apoptosis-inducing ligand, TRAIL/Apo2L, using TRAIL/Apo2L gene-deficient mice. Eur. J. Immunol. 32:2246-2254.

41. Masuzawa, T., et al. 1994. Estrogen-deficiency stimulates B lymphopoiesis in mouse bone marrow. J. Clin. Invest. 94:1090-1097.

42. Everts, V., et al. 2002. The bone lining cell: its role in cleaning Howship's lacunae and initiating bone formation. J. Bone Miner. Res. 17:77-90.

43. Parfitt, A.M. 2001. The bone remodeling compartment: a circulatory function for bone lining cells. J. Bone Miner. Res. 16:1583-1585.

44. Gregorio, G.B., et al. 2001. Attenuation of the self-renewal of transitamplifying osteoblast progenitors in the murine bone marrow by 17ß-estradiol. J. Clin. Invest. 107:803-812.

45. Kong, Y.-Y., et al. 1999. OPGL is a key regulator of osteoclastogenesis, lymphocyte development and lymph-node organogenesis. Nature. 397:315-323.

46. Kong, Y.-Y., et al. 1999. Activated T cells regulate bone loss and joint destruction in adjuvant arthritis through osteoprotegerin ligand. Nature. 402:304-309.

47. Yano, K., et al. 1999. Immunological characterization of circulating osteoprotegerin/osteoclastogenesis inhibitory factor: increased serum concentrations in postmenopausal women with osteoporosis. J. Bone Miner. Res. 14:518-527. 